\title{
A BEAT PHENOMENON IN DWARF NOVA ERUPTIONS
}

\author{
N. Vogt
}

European Southern observatory

Casilla 16317, Santiago 9, Chile

\section{Summary}

Photoelectric observations of the dwarf nova Vw Hyi, obtained at the end of the December 1975 supermaximum, are presented. After decline from the outburst, the superhump period (0\$07622) combines with the orbital period (Od07427) to a beat phenomenon: the O-C's and the light curves of the orbital hump vary systematically with the phase of the beat period for at least one week after recovery from the supermaximum. It is suggested that the red secondary component, which rotates non-synchroneously with the superhump period, expands slightly at the beginning of a supermaximum and is heated up asymmetrically, probably due to instabilities in its convection zone. In addition, the increased mass transfer rate may trigger the long eruption in the accretion disc while short eruptions originate in the disc without participation of the secondary.

\section{Introduction}

As already outlined in the preceeding paper (Schoembs, 1977) VW Hyi is characterized by two very distinct types of outbursts: short eruptions which repeat every 28 days in average and which last about 4 days, and long eruptions which occur every half year with a mean duration of two weeks. These "supermaxima" follow their mean period very regularly and can be predicted with an accuracy of +12 days. Normal maxima, on the other hand, occur at intervals between 8 and 50 days (Bateson, 1977). The two types of maxima are exclusive, there are no intermediate cases of outbursts.

The occurrence of the "superhumps" has been observed in three different supermaxima (Vogt, 1974; Warner 1975; Häfner et al.., $1977 \mathrm{~b})$. The superhumps repeat with a period of about 38 larger than the orbital period of VW HY1. The superhump period decreases, in the course of a supermaximum; periods and decrease rates at different supermaxima are identical within the error of observation. Normal maxima have smooth light curves which never show superhumps.

The quite distinct behaviour of $\mathrm{VW}$ Hyi during normal eruption and supermaxima as well as the absence of any intermediate outburst types lead to the conclusion that two different physical processes are responstble for the origin of short and long eruptions.

\section{The Supermaximum of December 1975}

All three supermaxima mentioned above were observed at the beginning and at the brightest parts of the outburst. The decline stage and the recovery to normal light were finally followed during and after the supermaximum of December 1975. These photoelectric observations were made with the $1 \mathrm{~m}$ telescope of the European Southern observatory, La Silla, in the blue light and in the high speed mode with a time resolution of 3 seconds. The result of the Fourier and power spectrum analysis of these data are given in Häner et al. (1977a).

Figure 1 shows the condensed light curves. The first night (Dec. 21) the star was still near maximum light at $\sim 10^{\mathrm{m}}$. From earlier super maxima we know that at this stage the superhump is still visible. This 
enables us to identify the peaks as superhumps. Their time interval coincides exactly with the superhum period at the end of earlier supermaxima. The next night (Dec. 22) the star is declining rapid$l_{y}$ at a level of about $12^{\mathrm{m}}$. There are several periodic features in the light curve which are still repeating with the superhum period and not with the orbital period. On Dec. 23 the star has arrived at $13^{\mathrm{m}}$ near 1 ts minimum light. The orbital hump is present with quite high amplitude. A surprising behaviour, however, begins the following night, on Dec. 24 : the orbital hump has a completely distorted shape, which repeats from cycle to cycle, and which has a large deviation in $0-C$ of about 23 minutes, which corresponds to 208 of the entire orbital periodl Also in the following nights, there are systematic deviations $O-C$ and systematic changes of the hump shape.

\section{The Beat Phenomenon}

There is a strong evidence that we are observing here a beat phenomenon between the orbital period (odo7427) and the superhump period just at the end of the outburst (0.07622). In this case, the observed o-C deviations should vary systematically with the phase of the beat period $(38.1$ cycles $=2.83$ days). Indeed Fig. 2 shows a clear systematic trend. The curve was constructed under the assumption that the mean $O-C$ over a complete beat period should be zero, no secular changes are accepted. This assumption leads to an asymmetric beat curve whose rising branch is covered quite well with observations while its falling branch is hypothetical. The mean beat phases of the relevant observing nights are as follows:

$\begin{array}{lrrrrrr}\text { Date Dec. } 1975 & 23 & 24 & 25 & 26 & 29 & 31 \\ \text { Beat phase } & 0.04 & 0.36 & 0.72 & 0.07 & 0.11 & 0.83\end{array}$

Comparing the beat phases with their corresponding light curves as shown in FIg. I it turns out that not only the $0-C$, but also the shape of the hump varies systematically with the beat phase; near phase 0 (Dec. 23, 26 and 29), when superhump and orbital hump colncide, we observe more or less symmetric humps with a large amplitude and small deviations O-C. Near phase 0.4 (DeC. 24) the hump is broader, with smaller amplitude and extremely distorted. Finally, near phase 0.8 (Dec. 25 and 31 ) the O-C deviations and the hump shape are opposite to those at phase 0.4 . In spite of the fact that there was a lapse of 6 days between the two observations of the similar phases near 0.8 the main characteristics of the two light curves repeated with high accuracy (e.g., the "standstill" at the rising branch). This is an additional support to my interpretation of this behaviour as a beat phenomenon.

\section{The Secondary Triggers Supermaxima}

The most simple explanation of a beat phenomenon is the combination of two light sources that are modulated with different periods. One of the light sources is the hot spot on the accretion disc which is visible mainly at minimum light and follows the orbital period. The other source has to follow the superhump period. A reasonable location for this light source would be the surface of the red secondary companion, assuming that this star rotates with the superhump period. In order to account for the decrease of the superhump period in the course of a supermaximum we have to postulate that the secondary component expands rapidly for about io of its radius just at the beginning of a supermaximum. The angular momentum conservation leads to a longer rotation period. In the course of the supermaximum the secondary contracts slowly to its normal radius and as a consequence its rotation velocity increases. 


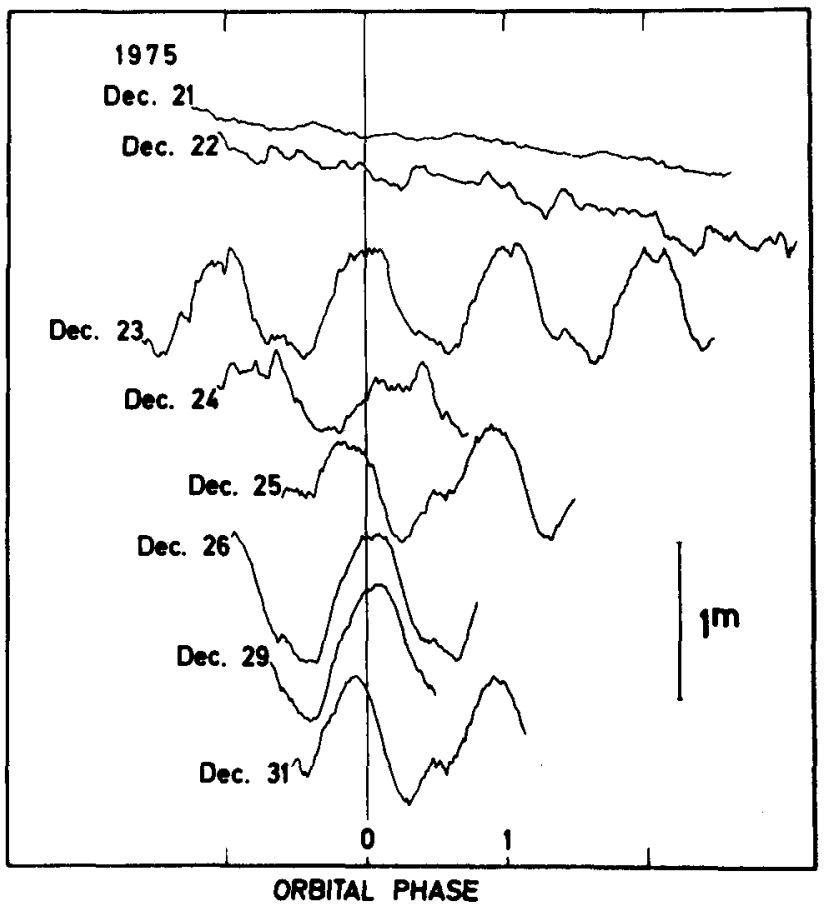

Fig. 1. Light curves of VW Hyi, 1975 Dec. 21-31

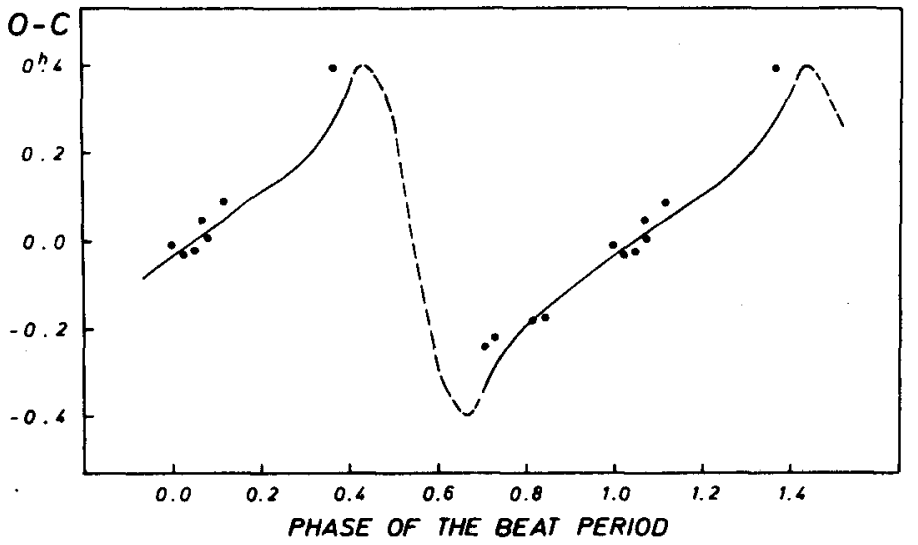

Fig. 2. Deviations of the observed hump times from the orbital ephemeris vs. phase of the beat period ( 38.1 cyles $=2.83$ days) 
The rapid expansion of the secondary at the beginning of the supermaximum will cause a suddenly increased mass transfer rate, and consequently, a strong disturbance of the stellar atmosphere of the secondary. This results in the formation of a hot area on that part of the stellar surface which has past the inner lagrangian point at the moment of the initial rapid mass transfer. Once the hot area is established, its periodic transit due to stellar rotation will cause the superhump phenomenon.

In spite of the increased luminosity of the red star, the main light source will be the accretion disc and not the hot area on the secondary. Apparently, this luminosity relation changes during the rapid decline, when the disc is fading faster than the secondary surface, as observed on Dec. 23. The spotty atmospheric structure of the red component remains stable for at least one week after decline and causes the beat phenomenon in combination with the hot spot of the disc in minimum light.

Nearly all recent publications attribute the origin of dwarf nova outburst to instabilities of the accretion disc or of the white dwarf (Warner, 1976, and references therein). In these models, the secondary plays a very passive role. On the other hand Gorbatsky (1975) gave arguments in favour of a sudden expansion and temperature increase of the secondary to be the origin of dwarf novae outbursts. He attributes the heating of the red star to instabilities in its convection zone. In a non-synchroneously rotating component of a close binary system the energy flux transported by convection may be lowered due to variations in gravity (Ivanov, 1972). Therefore, the energy produced by internal sources may be stored temporarily near the inner boundary of the convection zone. The time scale of the growth of this instability is of the order of $10^{7} \mathrm{sec}$ or 120 days, very near the typical cycle length of supermaxima.

5. Comparison with the Model of Schoembs

This paper represents an alternative to the model of schoembs (1977) as given in the preceeding paper. Common in the two models is the idea that the superhumps are caused by a non-synchroneously rotating spot on the red component which is partially heated up. The cause of this temperature increase is controversial: Schoembs adopts a mass flow from the disc to the red star. In my model instabilities, probably within the convection zone of the red star, result in a slight expansion and asymmetric heating-up of the atmosphere. Both models explain the superhump as well as the beat phenomenon after decline from supermaximum. Schoembs' model may be better compatible with some observational details, for instance the relatively late appearance of the superhump on the rising branch of the supermaximum. On the other hand, Schoembs gives no reason for the existence of the two distinct types of outbursts. My model contains a quite natural explanation for this, assuming that the short eruptions originate in the disc without direct participation of the secondary; long eruptions, on the other hand, are triggered by the secondary. 


\section{References}

Bateson, F.M. 1977: New Zealand Journal of Science 20, 73.

Gorbatsky, V.G.: 1975 in IAU Symposium No. 67, Variable Stars and Stellar Evolution, eds. V.E. Sherwood and L. Plaut, Reidel, Dordrecht, Holland, page 357.

Häfner, R., Schoembs, R. and Vogt, N.: 1977a: submitted to Astron. \& Astrophys.

Häfner, R., Schoembs, R. and Vogt, N.: 1977b: (in preparation).

Ivanov, L.N.: 1972, Vestnik Leningr. Univ. No. 13, 126.

Schoembs, R.: 1977, IAU Colloquium No. 42 (preceeding paper).

Vogt, N.: 1974, Astron. \& Astrophys. 36, 369.

Warner, B.: 1975, Monthly Notices Roy. Astron. Soc. 170, 219.

Warner, B.: 1976 in IAU Symposium No. 73, Structure and Evolution of Close Binary Systems, eds. P. Eggleton, S. Mitton, and J. Whelan, Reidel, Dordrecht, Holland, page 85 .

DISCUSSIO N of papers by SCHOEMBS and VOGT:

SHAVIV: How did you get a spot, or a spot-ring? If the disk becomes unstable it will blow-up to try and become spherical. I do not understand your objection to the spot in the disk. The point is that stability calculations show that the rise time of the instability is about $10^{2}$ revolutions.

The instabilities in the companion are due to the very low $\mathrm{g}$ near the surface. It is similar to what one finds in red giants. However, hydrodynamic calculations of these instabilities show that they are not periodic but rather irregular. This fact poses a problem to models that attribute the instability to the companion.

VOGT: I followed the possible trajectory of the matter. With sufficiently low velocity matter can fall back unto the companion.

KIPPENHAHN : After having listened to the presentations by Dr. Schoembs and Dr. N. Vogt, I must say that I am a bit confused, and I would appreciate if one of the two contributors could confront the two papers with each other in order to make it more easy for us to understand what they have in common, and where the points of view disagree.

SCHOEMBS: In Schoembs" model (Fig. 1) we have 1) Sudden and short $\left(<1^{d}\right)$ ejection of matter from an inner disc region at the beginning of a long eruption.

2) Only matter ejected into low inclination relative to the disc plane and into a certain interval of azimut can reach the secondary forming two bright spots symmetrical to the equator.

3) The spots follow the retrograd $\left(\mathrm{P}_{1}>\mathrm{P}_{0}\right)$ differential velocity field $P_{s}$ which depends on the latitude being more synchronized on the equatior. A meridional velocity component is assumed, 
moving the spot to the equator so that $\dot{\mathrm{P}}_{1}<0$.

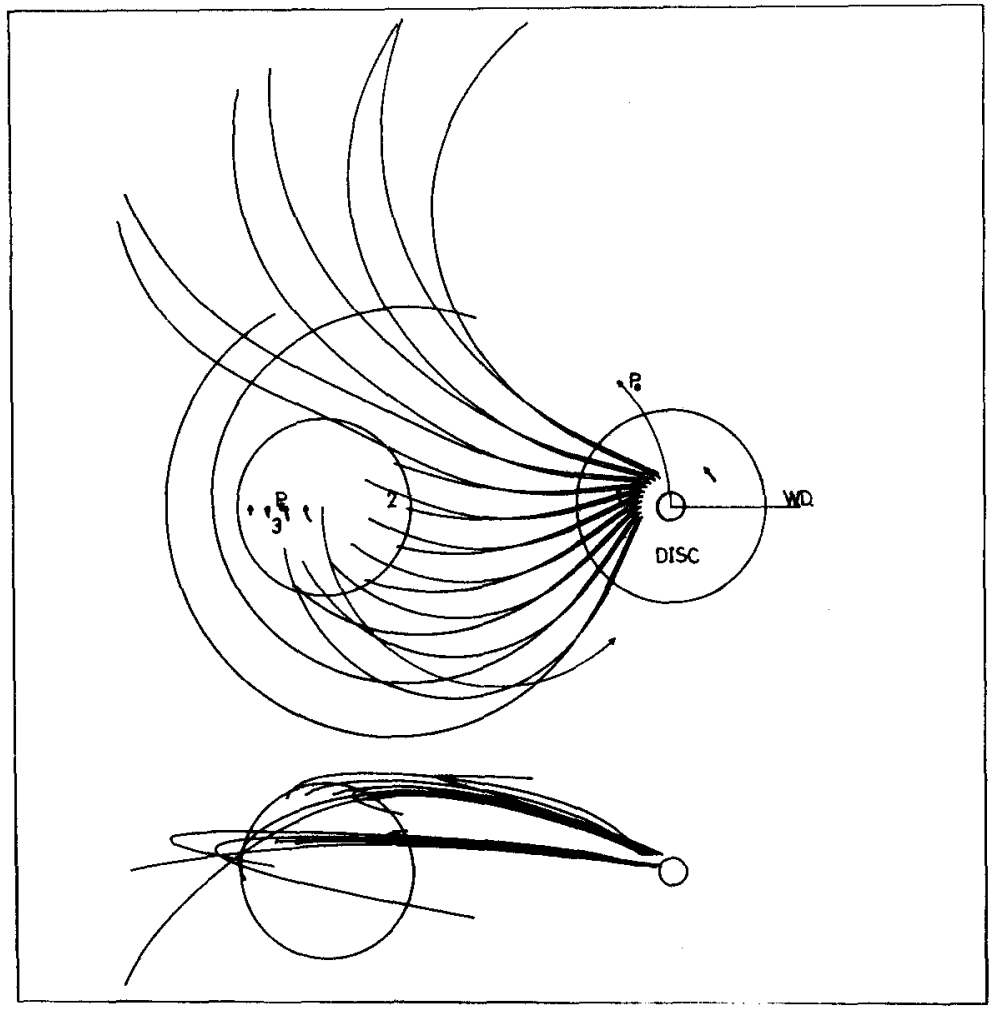

Fig.1. Computed trajectories for a radial initial expansion velocity $\mathrm{v}$ of $10^{\circ}$ and $30^{\circ}$ inclination aditional to the Keplerian velocity $v_{\mathrm{f}}$ in the disc at the beginning of the orbit. $v_{3}=1.1 v_{1} \cdot X Y$ plane top. $X Z$ plane bottom. The deformation of the secondary is not shown.

In Vogt's model we have (Fig. 2):

1) Slight expansion of the secondary by about 18 .

2) Increased mass flow to the disc triggering the luminosity increase.

3) Formation of a hot spot at the region facing the primary.

4) Due to prior expansion the spot rotates retrogradly. $\left(\mathrm{P}_{1}>\mathrm{P}_{0}\right)$. The following slow contraction causes the decrease of the period $\mathrm{P}_{1}\left(\dot{\mathrm{P}}_{1}<0\right)$. 
233

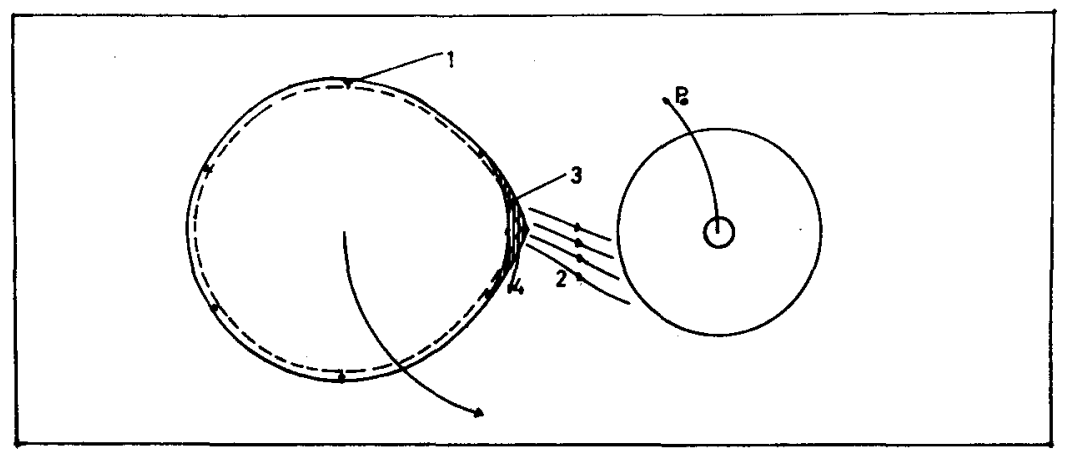

Fig. 2. Schematic drawing indicating the changes in the VW Fyi system during a long eruption.

https://doi.org/10.1017/S0252921100069396 Published online by Cambridge University Press 